\title{
Identification of Anthocyanins in Red Grape, Plum and Capulin by MALDI-ToF MS
}

\author{
Araceli Castañeda-Ovando, ${ }^{1 *}$ Ondra Sedo, ${ }^{2}$ Josef Havel, ${ }^{2}$ Lourdes Pacheco, ${ }^{1}$ Carlos Andrés Galán-Vidal, ${ }^{1}$ \\ and Elizabeth Contreras López ${ }^{1}$ \\ 1 Área Académica de Química. Universidad Autónoma del Estado de Hidalgo, Carr. Pachuca-Tulancingo, km 4.5 Mineral de la \\ Reforma, Hidalgo, México. ovandoa@uaeh.edu.mx \\ 2 Analytical Chemistry Department, Masaryk University, Kotlarska 2, 611 37, Brno, Czech Republic
}

Received December 6, 2011; Accepted June 15, 2012

\begin{abstract}
A simple and fast method was developed to identify anthocyanins in red grapes, plums and capulins by MALDI-ToF MS. Three different matrices were used: $\alpha$-CHCA ( $\alpha$-cyano-4-hydroxycinnamic acid), THA (2',4',6'-trihydroxyacetophenone) and DHBA (2,5-dihydroxybenzoic acid). Post-Source Decay (PSD) fragment detection allowed studying the structure of compounds detected. Deprotonated anthocyanin species were assigned in capulin ([Cyanidin-3-glucosidedeprotonated] $\mathrm{K}^{+}$and [Cyanidin-3-rutinoside-deprotonated] $\mathrm{K}^{+}$), due to these compounds also can be ionized in form of potassium adducts combined with a deprotonation.
\end{abstract}

Key words: MALDI-ToF MS, Grapes, Plums, Capulins, Deprotonated Anthocyanins.

\section{Introduction}

Anthocyanins are an important group of natural dyes, which belong to a larger class of plant chemicals known as flavonoids and are compounds with two aromatic rings, bound by a structure of three carbons and substituted with one or two sugar moieties. In Table 1 are shown the most frequent anthocyanidins (aglycons), and when $R_{1}$ is a sugar fraction (with or without acylated substitution) they are anthocyanins.
Resumen. Se desarrolló un método simple y rápido para identificar antocianinas en uvas rojas, ciruelas y capulines por MALDI-ToF MS. Se utilizaron tres matrices diferentes: $\alpha$-CHCA (ácido $\alpha$-ciano-4-hidroxicinámico), THA (2',4',6'-trihidroxiacetofenona) y DHBA (ácido 2,5-dihidroxibenzoico. La detección del fragmento por Decaimiento Post-Fuente (PSD) permitió el estudió de los compuestos detectados. En el capulín se asignaron especies desprotonadas de antocianinas ([3glucósido-de cianidina desprotonada] $\mathrm{K}^{+}$y [3-rutinósido de cianidina desprotonada $] \mathrm{K}^{+}$) ya que estos compuestos también pueden ionizarse en forma de aductos de potasio combinados con una desprotonación. Palabras clave: MALDI-ToF MS, uvas, ciruelas, capulines, antocianinas desprotonadas.

Models of anthocyanic pigments exhibited for the different species of fruits have demonstrated to be interesting for the chemotaxonomic researchers and nowadays they are frequently used for the control of mixtures or juices adulteration [1]. Additionally, the beneficial health effects and antioxidant properties $[2,3]$ of anthocyanins have generated a big interest in the research of anthocyanins during the last decade [2].

Anthocyanins represent the main water soluble pigments, they are directly responsible of the shiny orange, pink, red,

Table 1. Structural identification of the most common anthocyanidins (aglycons).

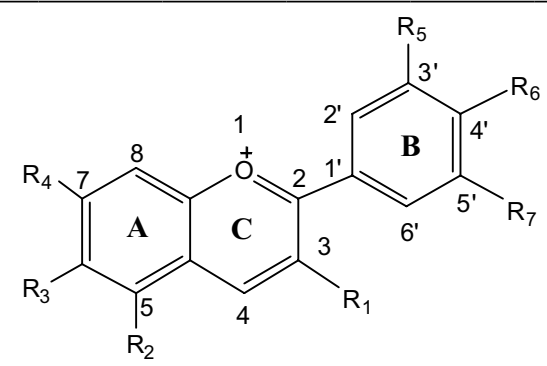

General anthocyanins structure

\begin{tabular}{lcccccccc}
\hline Name & Abbreviation & $\mathrm{R}_{1}$ & $\mathrm{R}_{2}$ & $\mathrm{R}_{3}$ & $\mathrm{R}_{4}$ & $\mathrm{R}_{5}$ & $\mathrm{R}_{6}$ & $\mathrm{R}_{7}$ \\
\hline Cyanidin & $\mathrm{Cy}$ & $\mathrm{OH}$ & $\mathrm{OH}$ & $\mathrm{H}$ & $\mathrm{OH}$ & $\mathrm{OH}$ & $\mathrm{OH}$ & $\mathrm{H}$ \\
Delphinidin & $\mathrm{Dp}$ & $\mathrm{OH}$ & $\mathrm{OH}$ & $\mathrm{H}$ & $\mathrm{OH}$ & $\mathrm{OH}$ & $\mathrm{OH}$ & $\mathrm{OH}$ \\
Malvidin & $\mathrm{Mv}$ & $\mathrm{OH}$ & $\mathrm{OH}$ & $\mathrm{H}$ & $\mathrm{OH}$ & $\mathrm{OMe}$ & $\mathrm{OH}$ & $\mathrm{OMe}$ \\
Pelargonidin & $\mathrm{Pg}$ & $\mathrm{OH}$ & $\mathrm{OH}$ & $\mathrm{H}$ & $\mathrm{OH}$ & $\mathrm{H}$ & $\mathrm{OH}$ & $\mathrm{H}$ \\
Peonidin & $\mathrm{Pn}$ & $\mathrm{OH}$ & $\mathrm{OH}$ & $\mathrm{H}$ & $\mathrm{OH}$ & $\mathrm{OMe}$ & $\mathrm{OH}$ & $\mathrm{H}$ \\
Petunidin & $\mathrm{Pt}$ & $\mathrm{OH}$ & $\mathrm{OH}$ & $\mathrm{H}$ & $\mathrm{OH}$ & $\mathrm{OMe}$ & $\mathrm{OH}$ & $\mathrm{OH}$ \\
\hline
\end{tabular}


magenta, violet and blue colors in the flower petals, in fruits as strawberries, capulin, plums, grapes, apples, cherries, and grasses $[4,5]$. Anthocyanic content for red grape, plum and capulin is shown in Table 2.

Mass Spectrometry is an analytical method used for anthocyanins identification, especially tandem with separation systems, such as liquid chromatography (LC), gas chromatography (GC) and capillary electrophoresis (CE).

Several ionization methods by desorption have developed in the two last decades, they used for non volatile or thermodynamically unstable samples, for example, the anthocyanins. These techniques dispense with volatilization and subsequent ionization, instead supplies power to solid or liquid sample in different ways, so that causes the direct formation of gaseous ions; obtaining simplified mass spectra.

Among these desorption sources are fast-atom bombardment (FAB), desorption electrospray ionization (DESI) and Matrix-assisted laser desorption ionization (MALDI), which are considered soft ionization sources, because they give rise to little fragmentation, providing useful information that allows accurate molecular weight determination of the molecule or molecules.

With respect to the use of Matrix-assisted laser desorption ionization-time of flight mass spectrometry (MALDI-ToF MS) for the anthocyanins analysis, speed of the analysis (approximately 4 minutes per sample) has been found as a main advantage [15].

Therefore, in this study was carried out the anthocyanins extraction from red grapes (Vitis vinifera), plums (Prunus domestica) and capulins (Prunus serotina); and were identified by the technique of MALDI-ToF MS with the purpose of developing a fast procedure to analyze these compounds.

\section{Results and discussion}

\section{Matrix selection}

All extracts were analyzed by MALDI-ToF MS using three different matrices. Besides previously proposed matrix THA, which was found to give good spot-to-spot repeatability for anthocyanin signals in wine extracts [16], $\alpha$-CHCA was selected as a matrix known for high ionization sensitivity and DHBA as a universal matrix capable to ionize various classes of compounds. The ability of the instrumentation to perform Post-Source Decay (PSD) fragment detection allowed to study the structure of compounds detected.

As the optimal matrix, $\alpha$-CHCA was selected yielding 10 fold higher signals of the analytes than DHBA or THA. Molecular weights of the compounds seen from the mass spectra correspond to a variety of anthocyanin derivatives.

Figure 1 shows mass spectra of the red grapes extract with three matrices used in the analysis ( $\alpha$-CHCA, DHBA and THA); the best signals were obtained with $\alpha$-CHCA matrix, and THA gave lower signal intensities.
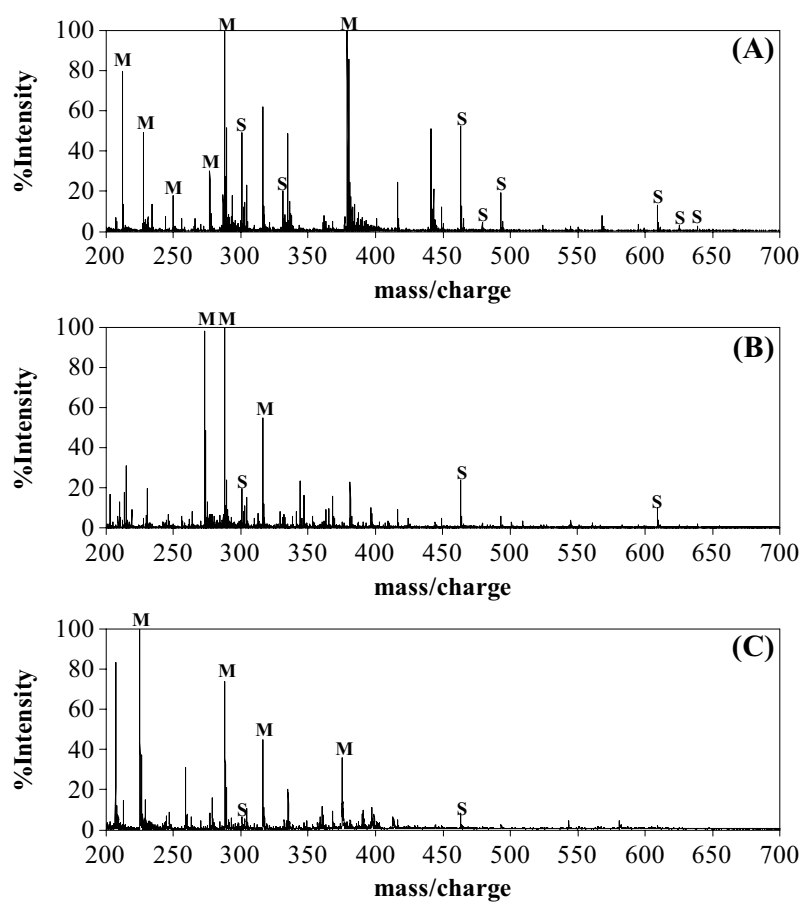

Fig. 1. MALDI-ToF mass spectra of red grapes extract obtained in reflectron positive mode with pulsed extraction, laser power of 2.83 $\mathrm{mW}$, and as matrix: (A) $\alpha$-CHCA, (B) DHBA, and (C) THA. Where: $\mathrm{M}$, signal for matrix; and $\mathrm{S}$, signal for sample.

Table 2. Anthocyanic content in red grape, plum and capulin.

\begin{tabular}{cl}
\hline Fruit & \multicolumn{1}{c}{ Anthocyanins } \\
\hline Red grape & 3-glucoside (Delphinidin, Cyanidin, Peonidin, Malvidin) [4,5-11] \\
& 3-acetil-glucoside (Malvidin, Delphinidin, Petunidin, Peonidin, Cyanidin) \\
& and 3-coumaril-glucoside (Malvidin, Delphinidin, Cyanidin, Petunidin, Peonidin) [7-10] \\
& 3,5-diglucoside (Delphinidin, Petunidin) [8] \\
& 3-caffeoyl-glucoside (Peonidin, Malvidin) [8,9] \\
& Cyanidin-3-sophoroside [4] \\
Plum & Cyanidin (3-glucoside, 3-rutinoside) [12,13] \\
& Peonidin (3-glucoside, 3-rutinoside) [13] \\
Capulin & Cyanidin-3-glucoside, Cyanidin-3-rutinoside [14]
\end{tabular}




\section{Red grapes}

The spectrum from red grapes, using $\alpha$-CHCA as matrix, is shown in Figure 2, signals were found at the $m / z$ values: 212.0, 227.9, 249.8, 277.1, and 288.1 ( $\alpha$-CHCA matrix cluster), 301.1 (Peonidin), 331.2 (Malvidin), 463.1 (Peonidin-3-glucoside), 479.1 (Petunidin-3-glucoside), 492.8 (Malvidin-3-glucoside), 609.2 (Peonidin-3-glucoside- $p$-coumarate), 625.2 (Peonidin3,5-diglucoside), and 639.3 (Malvidin-3-glucoside- $p$-coumarate). The last identification was obtained by PSD of all of the compounds, which was carried out with increased laser power and ion gate set to the corresponding masses. Table 3 shows the main compounds with their respective MALDI mass and PSD fragment mass.

The most common anthocyanins in red grapes are acylated, this is the reason of the red wine stability which comes from a combination of diverse factors such as: structure, concentration, $\mathrm{pH}$, temperature and the presence of complexing agents [17].

One of the PSD spectra (from Peonidin-3-glucoside- $p$-coumarate) is displayed in Figure 3, where, beside the precursor ion, only one intense signal can be observed. The peak results from the cleavage of glycosidic bond, which was found to be characteristic for all anthocyanin derivatives. Such fragment monitoring significantly facilitates the identification of this

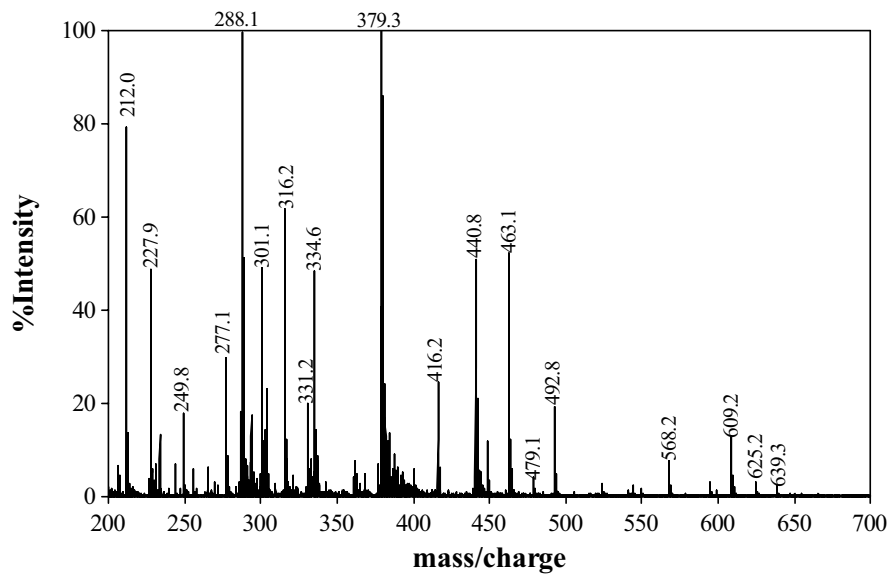

Fig. 2. MALDI-ToF mass spectrum of red grape extract obtained in reflectron positive mode with pulsed extraction and $\alpha$-CHCA as the matrix and laser power of $2.83 \mathrm{~mW}$. class of compounds, as the core of the anthocyanin molecule can be determined.

\section{Plums}

The spectrum from plums, using CHCA as matrix, is shown in Figure 4 . The peaks were observed at $\mathrm{m} / \mathrm{z}$ values: $228.0,335.7$ and 379.3 ( $\alpha$-CHCA matrix cluster), 287.2 (Cyanidin), 449.2 (Cyanidin-3-glucoside), 595.3 (Cy-3-glucoside- $p$-coumarate), and 611.5 (Cy-3-sophoroside). The main anthocyanins found in plums extract are derived from the cyanidin (Cyanidin-3-glucoside, Cy-3-glucoside- $p$-coumarate and $\mathrm{Cy}-3$-sophoroside), which are displayed in Table 4 with their respective MALDI mass and PSD fragment mass.

Last identification was obtained by PSD analysis of all of the compounds, with increased laser power and ion gate set to the corresponding masses. The spectrum of Cyanidin-3-sophoroside is presented in Figure 5, where, beside the precursor ion, only one intense signal can be observed.

\section{Capulins}

Capulins as plums presented anthocyanins derived from cyanidin, although with different substitution pattern (Table 5). The

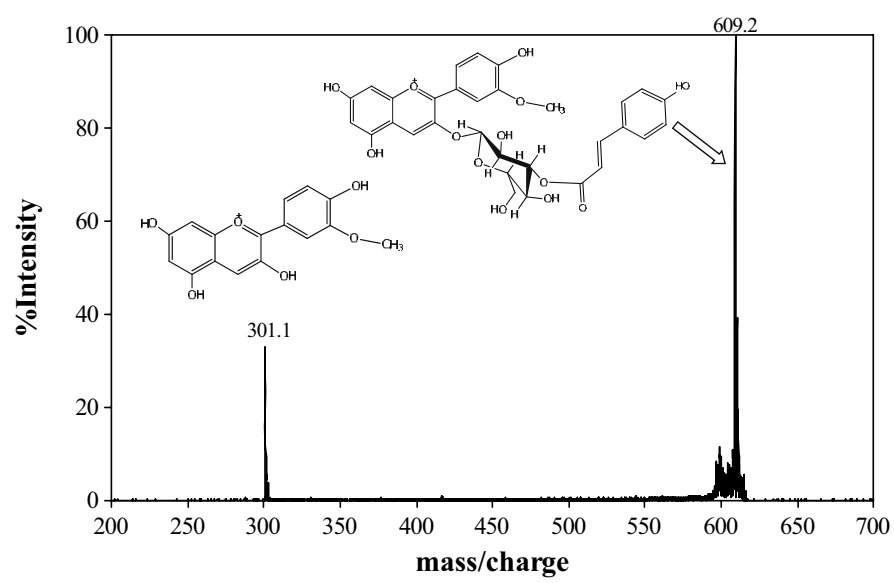

Fig. 3. PSD spectrum of Peonidin-3-glucoside- $p$-coumarate, obtained with ion gate set to $599-619 \mathrm{~m} / \mathrm{z}$ and laser power of $3.33 \mathrm{~mW}$.

Table 3. Main anthocyanins from red grape, $\alpha-\mathrm{CHCA}$ as the matrix.

\begin{tabular}{lccc}
\hline \multicolumn{1}{c}{ Compound } & MALDI mass, $m / z$ & PSD fragment mass, $m / z$ & $\%$ Relative intensity \\
\hline Peonidin & 301.1 & & 30.09 \\
Malvidin & 331.2 & & 12.36 \\
Peonidin-3-glucoside & 463.1 & 301.1 & 31.75 \\
Petunidin-3-glucoside & 479.1 & 317.1 & 2.39 \\
Malvidin-3-glucoside & 492.8 & 331.2 & 11.67 \\
Peonidin-3-glucoside- $p$-coumarate & 609.2 & 301.1 & 8.07 \\
Peonidin-3,5-diglucoside & 625.2 & 301.1 & 1.84 \\
Malvidin-3-glucoside- $p$-coumarate & 639.3 & 331.2 & 1.84 \\
\hline
\end{tabular}




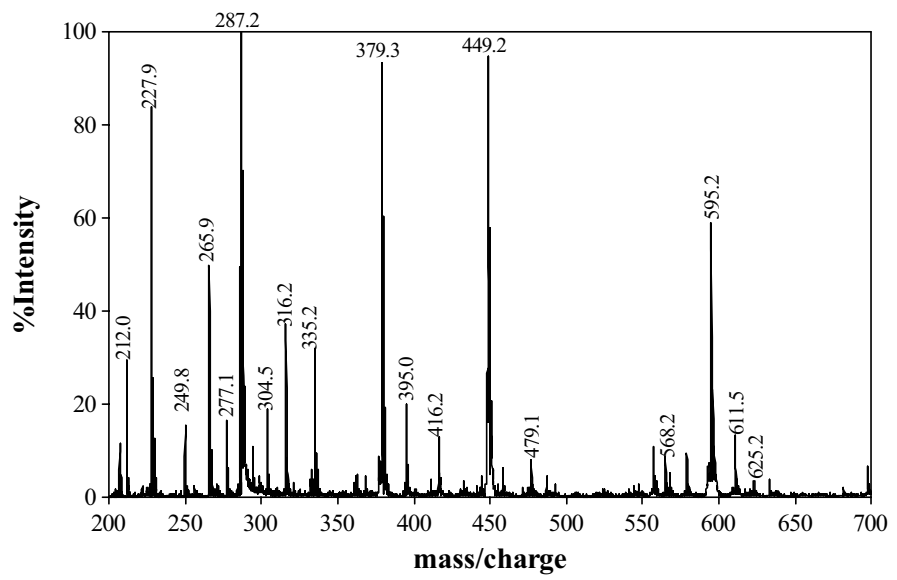

Fig. 4. MALDI-ToF mass spectrum of plum extract obtained in reflectron positive mode with pulsed extraction and $\alpha-\mathrm{CHCA}$ as the matrix and laser power of $2.83 \mathrm{~mW}$.

spectrum from capulin, using $\alpha-\mathrm{CHCA}$ as matrix, is shown in Figure 6 . The signals were found at the $m / z$ values: 416.7 and 568.2 ( $\alpha$-CHCA matrix cluster), 449.2 (Cyanidin-3-glucoside), 487.2 ([Cyanidin-3-glucoside-deprotonated] $\mathrm{K}^{+}$), 595.2 (Cyanidin-3-glucoside-p-coumarate) and 633.4 ([Cyanidin-3-rutinoside-deprotonated] $\mathrm{K}^{+}$).

PSD analysis was carried out with increased laser power and ion gate set to the corresponding masses. In Figure 7 is shown one of the PSD spectra (from [Cyanidin-3-rutinosidedeprotonated] $\mathrm{K}^{+}$).

Deprotonated species in capulin were assigned in function to spectra PSD, and according to references consulted, anthocyanins also can be ionized in form of potassium adducts combined with a deprotonation [18].

Peak in 325.2 (Figure 7), was assigned as rutinose, according with Ordaz-Galindo and col. [19] there is a pigment with $633 \mathrm{~m} / \mathrm{z}$, which they consider that it is a rutinoside derivate, as a result of the fragmentation in $325 \mathrm{~m} / \mathrm{z}$.
Such fragment monitoring significantly facilitates the identification of this class of compounds, as the core of the anthocyanin molecule can be determined. Therefore, PSD analysis of all observed species was found to be very useful and allowed almost complete extract mass spectra interpretation. For example, cyanidin-3,5-diglucoside and delphinidin 3-glucoside$p$-coumarate have almost the same molecular weight (mass resolution over 20.000 would be required to distinguish them) and therefore, PSD or other fragmentation technique is necessary to identify them.

It is interesting to note that diglucosidic species yield only weak or almost no signals corresponding to the loss of a glucose unit as simultaneous cleavage of both glycosidic bonds is preferred.

It is possible to follow from Table 2 that red grapes contain anthocyanins different from the other examined fruits. Moreover, the presence of cyanidin-3,5-diglucoside for plum.

MALDI-ToF MS was found to be a rapid method for the identification of anthocyanins in fruit extracts. Intense anthocyanin signals can be observed in the mass spectra when $\alpha$ cyano-4-hydroxycinnamic acid is used as a matrix. Such signal intensity is sufficient for Post-Source Decay analysis of these compounds, which is proposed as a highly effective tool for ultimate identification of the anthocyanins.

\section{Experimental section}

\section{Reagents}

MALDI matrices 2,5-dihydroxybenzoic acid (DHBA), $\alpha$ cyano-4-hydroxycinnamic acid ( $\alpha$-CHCA) and 2', 4', $6^{\prime}$-trihydroxyacetophenone (THA) were purchased from Sigma Aldrich (Steinheim, Germany). Acetonitrile was obtained from Merck (Darmstadt, Germany). De-ionized water double distilled from a quartz apparatus of Haraeus Quartzschmelze (Hanau, Germany) was used to prepare the solutions. Hydrochloric acid

Table 4. Main anthocyanins from plums, $\alpha$-CHCA as matrix.

\begin{tabular}{lccc}
\hline \multicolumn{1}{c}{ Compound } & MALDI mass, $m / z$ & PSD fragment mass, $m / z$ & $\%$ Relative intensity \\
\hline Cyanidin & 287.2 & & 37.33 \\
Cyanidin-3-glucoside & 449.2 & 287.2 & 55.50 \\
Cyanidin-3-glucoside- $p$-coumarate & 595.3 & 287.2 & 22.11 \\
Cyanidin-3-sophoroside & 611.5 & 287.2 & 5.05 \\
\hline
\end{tabular}

Table 5. Main anthocyanins from capulin, $\alpha-\mathrm{CHCA}$ as matrix.

\begin{tabular}{lccc}
\hline \multicolumn{1}{c}{ Compound } & MALDI mass, $m / z$ & PSD fragment mass, $m / z$ & \% Relative intensity \\
\hline [Cy-3-rut-deprotonated] $\mathrm{K}^{+}$ & 633.4 & 325.2 & 13.45 \\
Cy-3-glc-p-coum & 595.2 & 287.2 & 2.42 \\
[Cy-3-glc-deprotonated] $\mathrm{K}^{+}$ & 487.2 & 287.2 & 36.53 \\
Cy-3-glc & 449.2 & 287.2 & 5.55 \\
Rut & 324.5 & & 4.05 \\
\hline
\end{tabular}




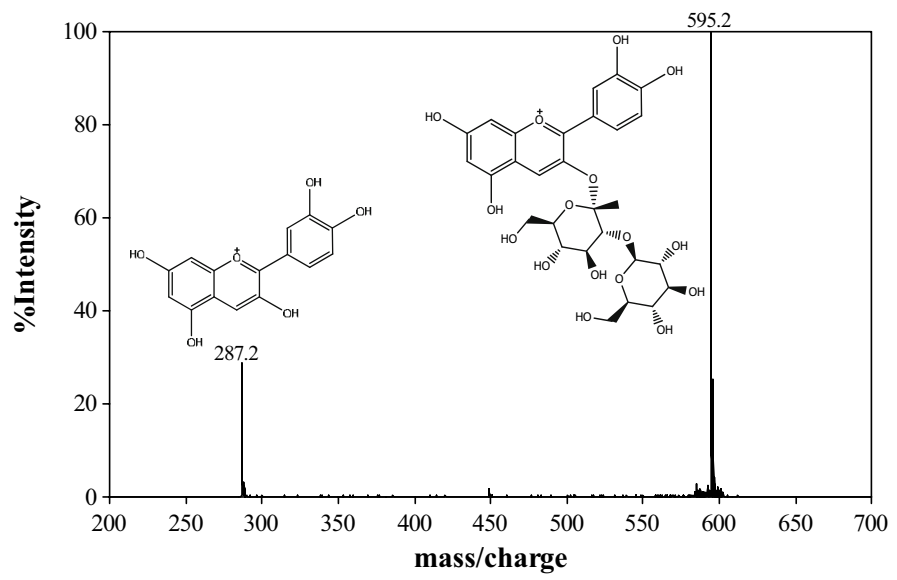

Fig. 5. PSD spectrum of Cyanidin 3-sophoroside, obtained with ion gate set to $585-605 \mathrm{~m} / \mathrm{z}$ and laser power of $3.33 \mathrm{~mW}$.

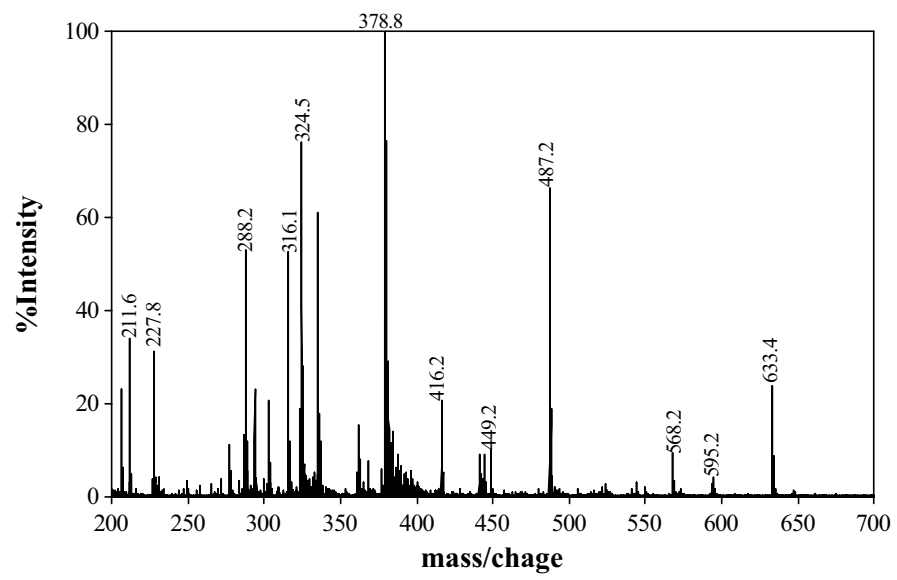

Fig. 6. MALDI-ToF mass spectrum of capulin extract obtained in reflectron positive mode with pulsed extraction and $\alpha$-CHCA as the matrix and laser power of $2.83 \mathrm{~mW}$.

was obtained from J. T. Baker (N. J., USA), methanol from Mallinckrodt (N.J., USA), and cartridges C18 high capacity from Alltech (Illinois, USA). All chemicals were of analytical grade purity. All fruits were bought in the supermarket.

\section{Extraction procedure}

The skins of approximately $1 \mathrm{~kg}$ of red grapes were extracted through sequential extraction with $200 \mathrm{~mL}$ of extractant $(2 \%$ (v/v) $\mathrm{HCl}-\mathrm{MeOH}$ ), during $2 \mathrm{~h}$. Four extractions were carried out. The same method was carried out with skins of plums (sample of $500 \mathrm{~g}$ ); for the sample of capulin, acetone was used as extractant, with the same procedure. Extracts were dried in a Büchi rotavapor at $30^{\circ} \mathrm{C}$ under reduced pressure until all residual extractant was evaporated. The extracts were dissolved in a small amount of methanol and they were purified through a cartridge $\mathrm{C} 18$ high capacity, $0.2 \%(\mathrm{v} / \mathrm{v}) \mathrm{HCl}-\mathrm{MeOH}$ was used as the eluent. The anthocyanic fractions were collected and dried in a Büchi rotavapor at $30{ }^{\circ} \mathrm{C}$ under reduced pres-

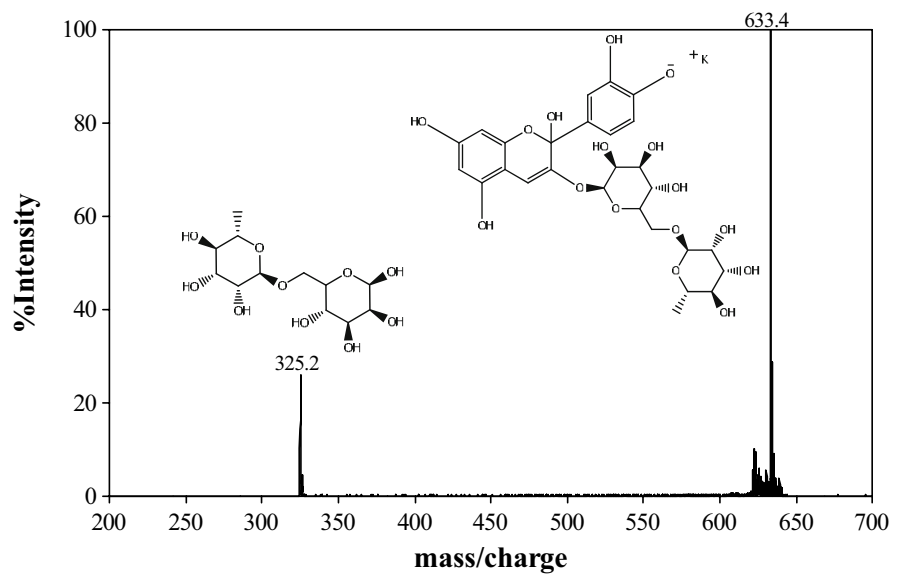

Fig. 7. PSD spectrum of Cy-3-rut-[deprotonated] $\mathrm{K}^{+}$, obtained with ion gate set to $623-643 \mathrm{~m} / \mathrm{z}$ and laser power of $3.33 \mathrm{~mW}$.

sure until all methanolic residual was evaporated. The extracts were dissolved in a small amount of deionized water and then lyophilized.

\section{Identification method}

MALDI-ToF mass spectra measurements were performed on Axima-CFR from Kratos Analytical, Shimadzu Group Company (Manchester, UK) equipped with nitrogen laser wavelength of $337 \mathrm{~nm}$ from Laser Science Inc. (Franklin, MA). All spectra were obtained as an accumulation of at least 100 laser shots. Matrix cluster peaks were used for internal mass calibration.

As matrices, saturated solutions of $\alpha$-CHCA or $30 \mathrm{mg} \mathrm{mL}^{-1}$ DHBA or THA in water:acetonitrile (1:1) were used. Extracts dissolved in methanol $\left(1 \mathrm{mg} \mathrm{mL}^{-1}\right)$ were applied on the sample plate in a volume of $1 \mu \mathrm{L}$ and immediately mixed pipetting up and down with $1 \mu \mathrm{L}$ of matrix solution. The mixture was then dried in an air stream.

\section{Acknowledgements}

Financial support from PROMEP (Project PROMEP/103.5/10/4407) is gratefully acknowledged. The authors acknowledged SNI (CONACyT) for the stipend received.

\section{References}

1. Obón, J. M.; Díaz-García, M. C.; Castellar, M. R. J. Food Compos. Anal. 2011, 24, 760-771.

2. Castañeda-Ovando, A.; Pacheco-Hernández, M. L.; Páez-Hernández, M. E.; Rodríguez, J. A.; Galán-Vidal, C. A. Food Chem. 2009, 113, 859-871.

3. Kahkonen, M.; Heinonen, M. J. Agric. Food Chem. 2003, 51, 628-633.

4. Goiffon, J. P.; Mouly, P. P.; Gaydo, E. M. Anal. Chim. Acta 1999, 382, 39-50. 
5. Fossen, T.; Slimestad, R.; Øvstedal, D. O. Andersen, O. M. Biochem. System. Ecol. 2002, 30, 855-864.

6. Saenz-Lopez, R.; Fernandez-Zurbano, P.; Tena, M. T. J. Chromatogr. A 2003, 990, 247-258.

7. Revilla, E.; Ryan, J. M.; Martín-Ortega, G. J. Agric. Food Chem. 1998, 46, 4592-4597.

8. Baldi, A.; Romani, A.; Mulinacci, N.; Vincieri, F. F.; Casetta, B. J. Agric. Food Chem. 1995, 43, 2104-2109.

9. Núñez, V.; Monagas, M.; Gomez-Cordovés, M. C.; Bartolomé, B. Postharvest Biol. Tec. 2004, 31, 69-79.

10. Heredia, F. J.; Francia-Aricha, E. M.; Rivas-Gonzalo, J. C.; Vicario, I. M.; Santos-Buelga, C. Food Chem. 1998, 63(4), 491-498.

11. Carreño, J.; Almela, L.; Martínez, A.; Fernández-López, J. A. Lebensm.-Wiss. u.-Technol. 1997, 30, 259-265.

12. Chun, O. K.; Kim, D. E.; Lee, C. Y. J. Agric. Food Chem. 2003, $51,8067-8072$.
13. Tomás-Barberán, F. A.; Gil, M. I.; Cremin, P.; Waterhouse, A. L.; Hess-Pierce, B.; Kader, A. A. J. Agric. Food Chem. 2001, 49, 4748-4760.

14. Ordaz-Galindo, A.; Wesche-Ebeling, P.; Wrolstad, R. E.; Rodriguez-Saona, L.; Argaiz-Jamet, A. Food Chem. 1999, 65, 201206.

15. Wang, J.; Kalt, W.; Sporns, P. J. Agric. Food Chem. 2000, 48, 3330-3335.

16. Wang, J.; Sporns, P. J. Agr. Food Chem. 1999, 47, 2009-2015.

17. Mazza, G.; Brouillard R. Phytochemistry 1990, 29, 10971102.

18. Wang, J.; Kalt, W.; Sporns, P. J. Agric. Food Chem. 2000, 48, 3330-3335.

19. Ordaz-Galindo, A.; Wesche-Ebeling, P.; Wrolstad, R. E.; Rodriguez-Saona, L.; Argaiz-Jamet, A. Food Chem. 1999, 65, 201206. 